\title{
Theoretical study of the interaction between vanadium oxide and HY-Zeolite elucidating loss activity of the catalyst
}

\author{
Irineo Pedro Zaragoza ${ }^{1, *}$, Maricela Arroyo ${ }^{2}$ and Benjamín Vargas Arista ${ }^{1}$ \\ ${ }^{1}$ Instituto Tecnológico de Tlalnepantla, División de Posgrado e Investigación, Av. Mario Colín S/N La \\ Comunidad, Tlalnepantla de Baz C.P. 54070, Estado de México, México \\ ${ }^{2}$ Instituto de Ingeniería, Universidad Nacional Autónoma de México, C.P. 04510. Ciudad de México
}

\begin{abstract}
The study of the interaction between vanadium oxide and the HY-zeolite using molecular dynamics interaction was carried out for two systems: i) when vanadium oxide penetrates a zeolite ring at its center, and ii) when vanadium oxide impacts the zeolite surface model. The dynamical effects are used to investigate the reduced activity and eventual degradation of the catalyst for the vanadium oxide presence. In the first case, we observe the breaking of an $\mathrm{OH}-$ bond that belongs to the acid site. This is related to an initial loss activity stage of the catalyst. In the second case, vanadium oxide is weakly adsorbed onto the zeolite surface velocity depending. Density functional theory, with nonlocal exchange and correlation functional and the basis set of double numerical accuracy, is used to analyze the electronic structure. It was used in combination with BornOppenheimer dynamics to perform calculations.
\end{abstract}

Keywords: HY-zeolite, vanadium oxide, density functional theory, molecular dynamics.

\section{Introduction}

The process for refining crude oil to get gasoline, better known as fluid catalytic cracking (FCC), is a technology currently in use. The use of solid catalysts such as zeolites in refineries produces an increment in the number of octanes in gasoline. Furthermore the heavy crude is a source of pollutants of nickel and vanadium, however, this catalyst shows a tolerance to contaminants 1 . The effects of metal contaminants as vanadium, nickel, sodium, iron are well known. The vanadium isotopes are presented in crude as organometallic complexes and porphyrins ${ }^{2}$.

In the regeneration of the catalyst within the FCC process to eliminate the coke and other contaminants from the surface it is noted that the vanadium is oxidized to $\mathrm{V} 2 \mathrm{O} 5$ and $\mathrm{VO}$ between other oxides. The $\mathrm{VO}$ phase has a NaCl-type structure and is unstable at ambient temperature, however, at regenerator conditions it was found that $\mathrm{V} 2 \mathrm{O} 5$ is fragmented into $\mathrm{VO}+\mathrm{VO} 4$ and the $\mathrm{VO}$ fragment is more reactive. Unlike other metals, vanadium migrates easily throughout the catalyst reducing its activity and selectivity by destroying zeolite crystallinity. It is important to understand the mechanism of zeolite destruction since it may help to stop the process of vanadium movement and to improve the stability of catalyst ${ }^{3,4}$.
Several mechanisms have been proposed for explaining the zeolite's collapsing process in presence of vanadium. Thermodynamic data analysis, suggest that $\mathrm{V} 2 \mathrm{O} 5$ reacts with water to form the vanadic acid $\mathrm{H} 3 \mathrm{VO} 4$ under high temperature conditions of oxidation, this acid can attack the $\mathrm{Si}-\mathrm{O}-\mathrm{Al}$ bond causing damage on zeolite structure. Also based on experiments it was found synergy between the destructive action of sodium and vanadium, indicating that without the presence of sodium, vanadium by itself has little effects on zeolite crystallinity. In the same way, the results of an experimental study of molecular structure of oxovanadium porphyrin concludes that the bonding related to zeolite could be by means of an oxygen atom of vanadyl group, which presents a tendency to form a bond with hydrogen coming from an acid site of zeolite ${ }^{5-7}$. Recently the effects of vanadium contamination on the framework and micropore structure of USY zeolite were studied ${ }^{8}$.

The main purpose of this study is to establish a procedure to explain the loss activity of a catalyst by specific physical properties through the dynamic interaction between the vanadium oxide and a fragment of zeolite HY. The forces are considered to explain the main types of breaking atomic bonds, nevertheless the existing weak force that is associated to the adsorption. For this purpose, an analysis, of the change of energy values in relation- 
ship with time scales for each model, is done. The vanadium oxide follows a preferential trajectory at each step, the forces on the atoms are computed and combined with the current position and the velocity generates new position and velocity a short time ahead. The degrees of freedom in the interaction between vanadium oxide and acid site allow a spontaneous trajectory that depends on forces. The forces that promote the bond dissociation are complex to determine in each time because all electrons are involved and are determined by the calculation of dynamics molecular applied the BornOppenheimer approximation at the level of the DFT theory.

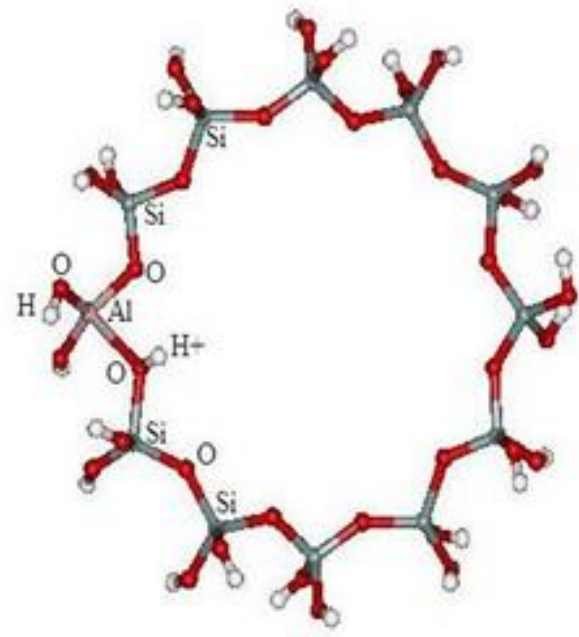

a)

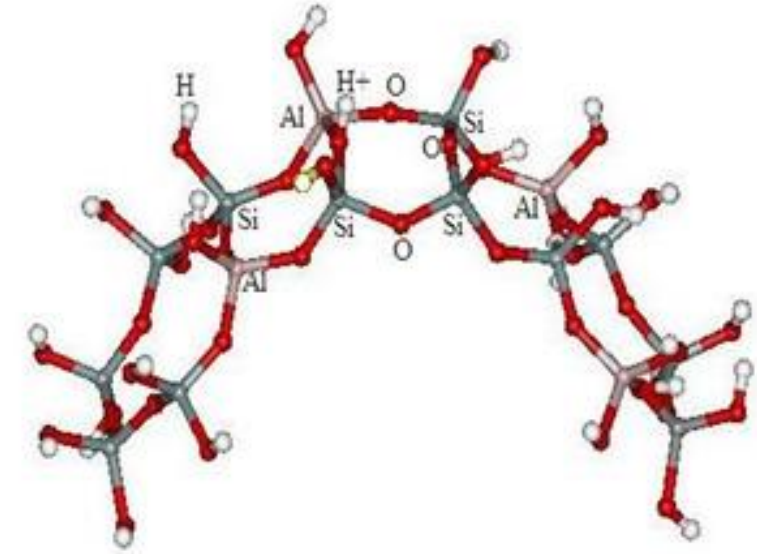

b)

Figure1. HY-zeolite models: a) ring with 12 tetrahedral sites; one of them is an acid site (Al-O- $\left.\mathrm{H}^{+}\right)$, b) crystallographic portion of the HY-zeolite surface.

\section{Model and Method}

The characterization of the HY-zeolite has been carried out by means of the joint use of different technics: neutron powder diffraction, infrared and mass spectroscopy. Take into account to know that HY-zeolite has structural conformation in each group of pores it is characterized by highly symmetric rings, such rings are constituted by a number of tetrahedral $(\mathrm{T})$ silicon $(\mathrm{SiO} 4)$ and aluminum $(\mathrm{AlO} 4)$ 9. The label Y of the zeolite is attributing to the fact that the HY-zeolite shows Y-shape ramifications. The $\mathrm{Al}$ sites are commonly identified as the acid sites $(\mathrm{AlOH}+)$ because it is at these places where a deficiency of electron charge occurs, with the consequent catalysis on hydrocarbons at such places 10-12. The acid sites located on the external surfaces of the HY-zeolite also reacted with hydrocarbons, usually larger than the ones traveling into nanopores. However, the catalytic sites at the external surface exhibit a greater propensity to be neutralized. In order to simulate the interaction of an exposed catalytic site with vanadium oxide, we have chosen two models. For the first one, we have taken a ring with 12 tetrahedral sites, one of them is an acid site; the internal diameter is around to 8 angstroms, (Fig.1) a). For the second one, we have chosen a crystallographic portion of the HY-zeolite surface. It consists of 16 T-sites as depicted in (Fig. 1) b). In four arbitrarily selected $\mathrm{T}$-sites the silicon atom was replaced by aluminum, in such a way that the zeolite composition was (SiO4)12(AlO4)4, with a Si:Al ratio of 3 , in agreement with the experimentally observed and stable Y-zeolites. In each oxygen bridge connecting the $\mathrm{Al}$ and $\mathrm{Si}$ atoms, $\mathrm{Al}$ attached a hydrogen atom to compensate the local charge perturbation produced by the replacement of $\mathrm{Si}$. The external surface of the zeolite models is saturated with hydrogen atoms bonded to oxygen atoms, keeping the bonding symmetry and at normal $\mathrm{O}-\mathrm{H}$ bond length.

The interaction between vanadium oxide and the zeolite surface is investigated applying Density Functional Theory (DFT) because it is appropriated to determine structural and electronic property changes with relatively high accuracy methods that include electronic correlation and spin-orbital corrections. The Density Functional Theory 13 (DFT), included in the NWChem software ${ }^{14}$, has been used in this case. The approximation method uses an exchange-correlation energy functional, the Non-Local Density Approximation (NLDA) with Becke $88{ }^{15}$ this is an exchange energy functional of the corrected gradient with asymptotic behavior, and Lee-Yang-Parr ${ }^{16}$ for correlation. When the nonlocal version of DFT is compared with the local approximation of DFT, it is found that the nonlocal approach gives an improved prediction of the electronic properties and interaction energies of molecules, due to its higher capacity to explain the anisotropy of the charge distribution ${ }^{17}$. In general, the nonlocal DFT results are found in good agreement with the experimental measurements ${ }^{18}$. The DZVP basis set was used in all calculation for all atoms. They were specially constructed for use with the DFT approach and exhibit small basis set 
superposition errors. However, to avoid extensive machine-time computational and other computational bottlenecks attributed to the employment of the DZVP basis sets, this basis set should not introduce artificial electronic distortions. The atoms that do not participate in the zeolite catalytic pocket are assumed to play a less important role in the interaction between oxide vanadium and the catalytic pocket due to their location relatively far away from the surface. This computational level has shown good results for molecules that contain vanadium, silicon, aluminium, oxygen and hydrogen atoms with a coarse grid ${ }^{19}$. A neutral charge for the whole system is considered; in this case, the spin multiplicity is 2 . The scheme used in the dynamics tasks is a quasi-Newton algorithm with line searches and approximate energy Hessian updates, also it is implemented in the NWChem. A maximum cartesian step of 0.0015 Bohr and a convergence of 0.0015 Hartree/Bohr in the energy gradients are conveniently chosen. The molecular dynamics simulation technique is important because it allows knowing the dynamical nature of the nuclear particles since they help to gain a deeper understanding of the mechanism of dissociation and binding bond reaction over the vanadium oxide and HY-zeolite atom system. These reactions are simulated by Born-Oppenheimer approximation method. The electronic wave function is obtained by taking into account that electrons are immersed in a field of instantaneously fixed nuclei, whereas the nuclear particle is immersed in an average electronic field. The heavy masses and very short spatial extension of the nuclear particles compared to those of electrons let us simplify the problem by considering the nuclei as a classical particle. In order to this happen, each nucleus follows a Newtonian trajectory due to quantum forces derived from the electron potential plus the electrostatic force exerted by the other nuclear particles. The time step in the dynamics is 1 fs. The self-consistent approach is considered finished when a convergence of $10^{-5}$ au in the energy and density are obtained. The atoms allowed to move freely in the molecular dynamics simulation are the same as these in the optimization process. The computations are performed in Laboratorio Nacional de Supercomputo del Sureste de México, BUAP.

\section{Results and Discussion}

In the vanadium oxide, there are two degrees of freedom to interact with the zeolite taking certain initial conditions. One of them is the position and the other the speed. In the position, there is a distance of around $10 \AA$ and gives a value to the initial speed. In the models of the zeolite, the same conditions apply. Dynamics calculations for interactions have been carried out using two models of interaction exploring all possible degrees of freedom.

\section{Interaction of the VO molecule on $\mathrm{HY}$-zeolite ring. \\ The first model is used to simulate the} interaction between $\mathrm{VO}$ and HY-zeolite and has taken a structure composed of 12 sites tetrahedral one of them is the acid site $\left(\mathrm{H}^{+} \mathrm{O}-\mathrm{Al}-\mathrm{O} 3\right)$ nearest to be allowed to freely move. The remaining oxygens, hydrogens and silicon atoms were frozen since, under the consideration that the catalysis usually exhibits a local character, they are located far from the catalytic pocket and can be considered as static or hold by a larger framework, also the vanadium oxide molecule is free to move. This model is used to calculate the electronic properties as part of the structural changes that are promoted in these dynamic interactions. In order to promote the dynamic interaction of the VO with freedom of the possible adsorption on the acid site was placed in the ring center of zeolite. These conformations may be involved in every step of the dynamic energy changes that are associated with the length of the atomic bonds. Also, presents important adsorption processes or possible reactions that can be involved in each step of dynamics related to energy changes that are associated to bond length atoms and position of atoms.

The DFT calculation was chosen to be unrestricted to spin calculation since VO is an open shell electronic system. After several cycles, the molecule approaches to acid site and it is observed that cationic hydrogen, $\mathrm{H}^{+}$, is the relevant site to characterize the force effect and this depends on the values of the initial speed. For the case when selecting the speed with the value of 3.0 the calculation results determined that the VO molecule does not interact with the acid site. For this reason, the values are decreased to 1.5 and the calculation determined that VO molecule interacts with the acid site. Fig 2 shows the energy changes as a function of time without temperature, but with an initial speed given to the molecule VO. Firstly, carried out calculation where the molecule VO is about to the HY-zeolite in the range of 0-190 fs getting a relationship of the values of positive energy. Then, the adsorption process occurs taking into account the negative values of energy in the range of 190-340 fs within this interval that adsorption is associated with the attractive force promoted by the $\left(\mathrm{O}_{3} \mathrm{AlOH}^{+}\right)$in 250-340 fs until minimum energy value of the -28 $\mathrm{kcal} / \mathrm{mol}$. In addition, the $\mathrm{VO}$ is placed near to tetrahedral site of the ring and takes a preferential pathway selecting the $\mathrm{OH}^{+}$until generating changes in the atomic bonds in the range of 340-365 fs there is an increase of $25 \mathrm{kcal} / \mathrm{mol}$ for the energy associated with the bond breaking of $\mathrm{O}-\mathrm{H}^{+}$and this appended to $\mathrm{VO}$ in the 365-395 with energy values of $28 \mathrm{kcal} / \mathrm{mol}$ to form bond (V-O-H+). Finally, in the 400-450 the atomic bonds showed the following changes in 
H-OV-O-Al-O with a maximum and minimum values of energy. These results are used to take a consideration of the interaction between $\mathrm{VO}$ and
HY-zeolite in a ring model that takes criterions of elucidating the loss activity of the type of catalyst in particular place and condition of calculation.

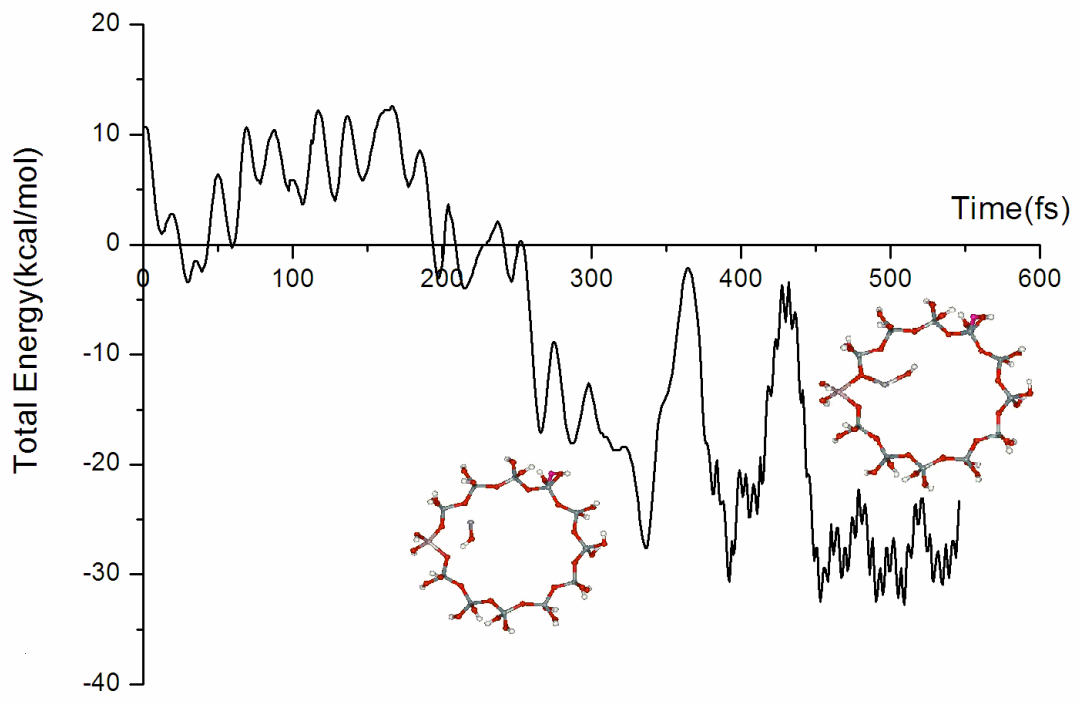

Figure 2. The total energy values in the interaction between VO with a HY-zeolite ring conformed by 12 different tetrahedral sites show two interesting moments, one of them is where the adsorption occurs and the second one is a result of the atomic exchange to generate new atomic bonds nearest to the acid site $\left(\mathrm{Al}-\mathrm{O}-\mathrm{H}^{+}\right)$by determining which values of energies exist during the simulation.

Table 1. The bond length values on the initial conformation and the bond breaking like modified due to interaction between $\mathrm{HY}$ zeolite and VO for ring model.

\begin{tabular}{|c|c|c|}
\hline $\begin{array}{l}\text { Bond length of fragment } \\
\text { HYzeolite ring }\end{array}$ & Initial $(\AA)$ & Final $(\AA)$ \\
\hline $\mathrm{Si}-\mathrm{O}-\mathrm{Al}-\mathrm{O}-\mathrm{H}^{+}$ & $\begin{array}{l}\mathrm{Si}-(1.64)-\mathrm{O}-(1.73)-\mathrm{Al}-(2.10)-\mathrm{O}-(0.98)- \\
\mathrm{H}^{+}\end{array}$ & $\begin{array}{l}\text { Si-(1.68)-O-(1.89)-Al-(1.81)-O-(3.09)- } \\
\mathrm{H}^{+}\end{array}$ \\
\hline $\mathrm{V}-\mathrm{O}-\mathrm{H}^{+}$ & V-(1.69)-O-(5.78)-H+ & $\mathrm{V}-(1.69)-\mathrm{O}-(0.92)-\mathrm{H}+$ \\
\hline
\end{tabular}

The changes in the values of bond length between atoms are reported in Table 1, where it is noted that the main changes develop at the acid site tetrahedral happening in the bonds $(\mathrm{Si}-\mathrm{O}-\mathrm{Al}-$ $\left.\mathrm{O}-\mathrm{H}^{+}\right)$observing a bond breaking in $\mathrm{O}-\mathrm{H}(0.98$ to $3.09 \AA)$ and the $\mathrm{H}^{+}$generated a new bond with VO (5.78 to $0.92 \AA$ ), it's an important result because the catalysis of the zeolite develops by the $\mathrm{H}^{+}$exchange with other hydrocarbons which indicates that when it loses the zeolite $\mathrm{H}^{+}$thus elucidating the loss activity of the catalyst.

\section{Impact of the VO molecule on Surface HY-} zeolite.

The second model is confirmed by 16 tetrahedral sites as zeolite surface to study the interaction with VO. In the initial condition the VO was placed at certain separation distance from the zeolite surface; when the VO is near to four tetrahedral sites of the surface, it takes a preferential pathway selecting the acid site until it attains the adsorption point between the oxygen of the acid site and the vanadium atom of VO molecule. This point is relationship with the first minimum of kinetic energy as shown in Fig 3. Another condition is to promote an impetus to $\mathrm{VO}$, with a speed of 3.0 ; in the calculation is noted that VO gets through four tetrahedral sites without overcoming the potential barrier so that the $\mathrm{VO}$ is ejected by HY-zeolite surface indicating that exists a repulsion effect and other important point is that bond length of VO has not been modified. To take into account the increase the speed of 5.0 in this calculation, it is observed that vanadium-oxygen $(\mathrm{H}-$ $\mathrm{O}-\mathrm{V}$ ) took a minimum bond length around of $\mathrm{H}^{+} \mathrm{OAlO} 3$ as a consequence it produces the adsorption of the VO.

In the same way, it was carried out the calculation with the speed of momentum of 6.0 and the calculation performed shows two important points at the time when the molecule is closer to the place of interest; within the range of 0-70 fs it is noted that the total energy is increased in the same way that the kinetic energy decreases indicating that the total energy of the system changes to potential energy for interaction and corresponds to a repulsive force until molecule braking happens. In the time 75 fs relationship with value of energy $110 \mathrm{kcal} / \mathrm{mol}$ has an interesting point since the two plots have the same values indicating that initial processing of adsorption 
of VO over HY-zeolite and the modification of atomic bonds. After that, there are two maximum values in the range of 75-170 fs in particular the VO promotes alteration in the atomic bond of HY-zeolite

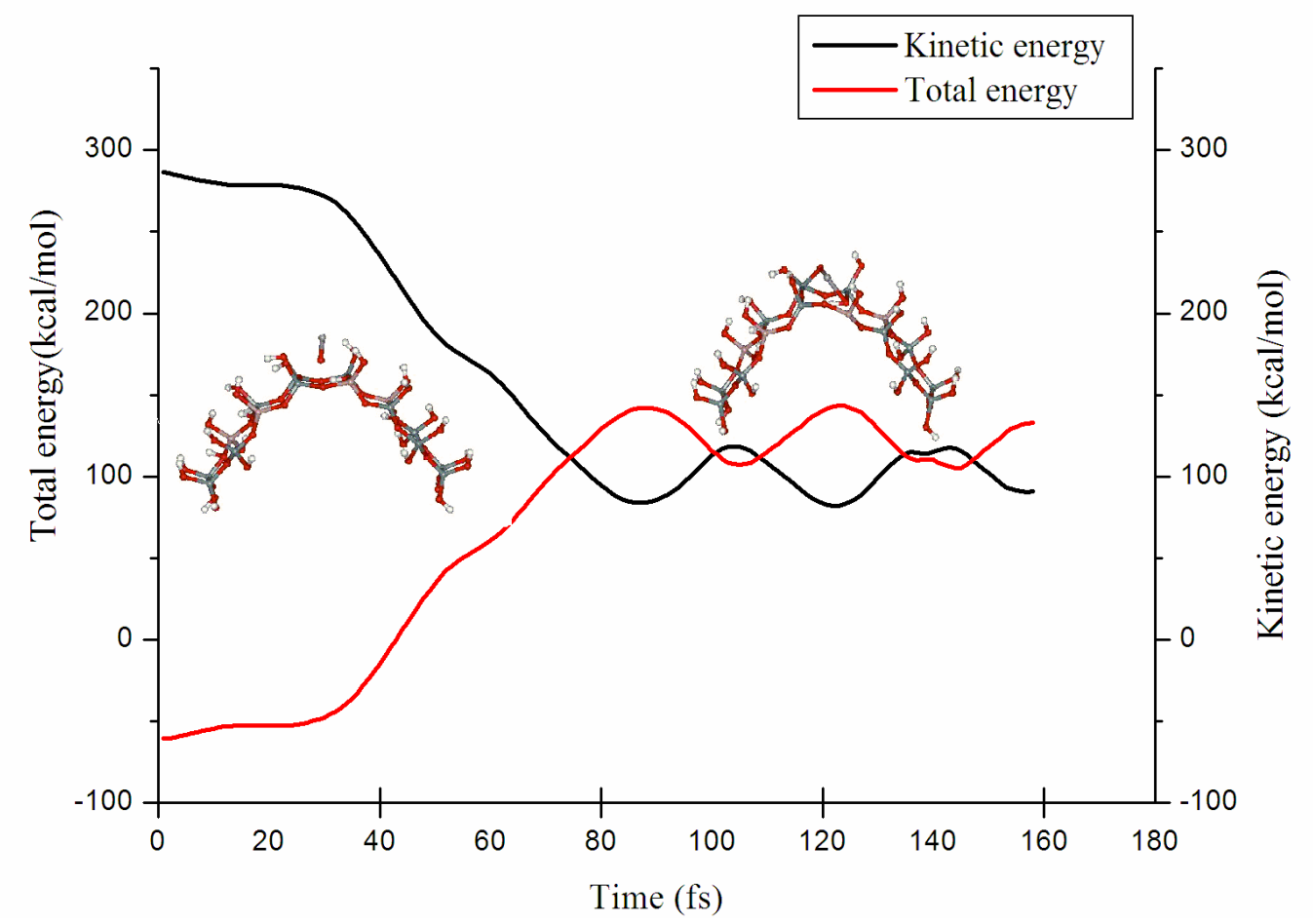

Figure 3. Total energy and kinetic energy are functions of time before and after the impact between the molecules that are described for each time of dynamic interaction around the minimum and maximum values as the main aspect of modifying the bond length of the acid site.

In Fig. 3 the plot shows how the values decrease to a minimum in $75-85 \mathrm{fs}$, after that, it has a behavior of maximum values in the period $85-105 \mathrm{fs}$, in the same description, minimum values of kinetic energy occurred in the 105-120 fs. In this calculation the dynamic interaction shows that the oxygen of vanadium oxide promotes a repulsive force with $\mathrm{H}^{+}$ and the $\mathrm{VO}$ rotates to take a singular perpendicular orientation to $\left(\mathrm{H}^{+} \mathrm{OAlO} 3\right)$, bond length values of $\mathrm{O}$ $\mathrm{V}$ and $\mathrm{H}+\mathrm{O}-\mathrm{Al}$ increase from 1.6 to $2.0 \AA$ and from 2.0 to $3.0 \AA$ respectively. These bond length modifications correspond to change in the values of total energy. The energy plot in Fig 3 shows that the speed allows that two molecules get closer until they collide due to the attractive force of the acid site with the oxygen of $\mathrm{VO}$ the bond of vanadium-oxygen changed of $1.6 \AA$ and $\mathrm{OH}^{+}$of $1.0 \AA$ ( $\left.\mathrm{V}-\mathrm{O}-\mathrm{H}^{+}-\mathrm{O}-\mathrm{Al}\right)$ took a minimum bond length around of $2.0 \AA$ at this adsorption point the bonds $(\mathrm{O}-\mathrm{H}-\mathrm{O}-\mathrm{V})$. The initial speed VO to allow interaction with HY-zeolite surface overcoming the repulsive potential that slows down until stopping it and at this moment acid site generates an attractive force. Fig 3 shows in the plot that minimum and the maximum value of the total energy is $40 \mathrm{kcal} / \mathrm{mol}$ that corresponds to bond length for the vanadium-oxygen and $\mathrm{OH}+$, affirming that the adsorption process is obtained.

Table 2. The bond length values on the initial conformation and the bond modified due to interaction between HY zeolite and VO for surface model.

\begin{tabular}{|c|c|c|}
\hline $\begin{array}{l}\text { Bond length of fragment } \\
\text { on surface HYzeolite }\end{array}$ & Initial $(\AA)$ & Final $(\AA)$ \\
\hline $\mathrm{Si}-\mathrm{O}-\mathrm{Al}-\mathrm{O}-\mathrm{H}^{+}$ & $\begin{array}{l}\mathrm{Si}-(1.66)-\mathrm{O}-(1.75)-\mathrm{Al}-(2.23)-\mathrm{O}-(0.99)- \\
\mathrm{H}^{+}\end{array}$ & Si-(1.76)-O-(1.81)-Al-(2.9)-O-(1.03)- $\mathrm{H}^{+}$ \\
\hline $\mathrm{V}-\mathrm{O}-\mathrm{H}^{+}$ & V-(1.60)-O-(2.95)-H+ & V-(2.06)-O-(2.46)-H+ \\
\hline
\end{tabular}

In the dynamics calculations, results for interaction between $\mathrm{VO}$ and $\mathrm{HY}$-zeolite were obtained interesting changes for the neighbors of the acid site ( $\mathrm{SiO} 4)$ connected by oxygen bridges to $(\mathrm{O} 3 \mathrm{AlOH}+)$. The values reported (Table 2) show an important modification; in the first row there are increases of $0.10 \AA$ in $(\mathrm{Si}-\mathrm{O}), 0.06 \AA$ in $(\mathrm{O}-\mathrm{Al}), 0.67$ in $(\mathrm{Al}-\mathrm{OH}+)$, and 0.04 in $(\mathrm{O}-\mathrm{H}+)$ all associated to $(\mathrm{Si}-\mathrm{O}-\mathrm{Al}-\mathrm{O}-\mathrm{H}+)$. The (VO) presence in the absorption region is relationship with the minima and maxima values that show an increase of 6 times more of elongation on the bond length $(\mathrm{Al}-\mathrm{OH}+)$ 
promoting a possible bond breaking. The adsorption of the vanadium oxide on the acid site also presents structural changes, as well as the distance of (VO) with $(\mathrm{H}+)$ represented as $(\mathrm{V}-\mathrm{O}-\mathrm{H}+)$, the value in the second row presents increases of $0.46 \AA$ in $(\mathrm{V}-\mathrm{O})$ showing an effect of elongation and possible bond breaking, there is an insignificant increase of $0.04 \AA$ of separation distance between (VO) and (H+) indicating that vanadium oxide does not affect proton of the acid site as it does with the tetrahedral sites $(\mathrm{AlO})$ and $(\mathrm{SiO})$. The $\mathrm{VO}$ is collocated over the acid site as shown Fig. 4 affecting the acid site in the activity of the zeolite and confirmed the VO adsorption.

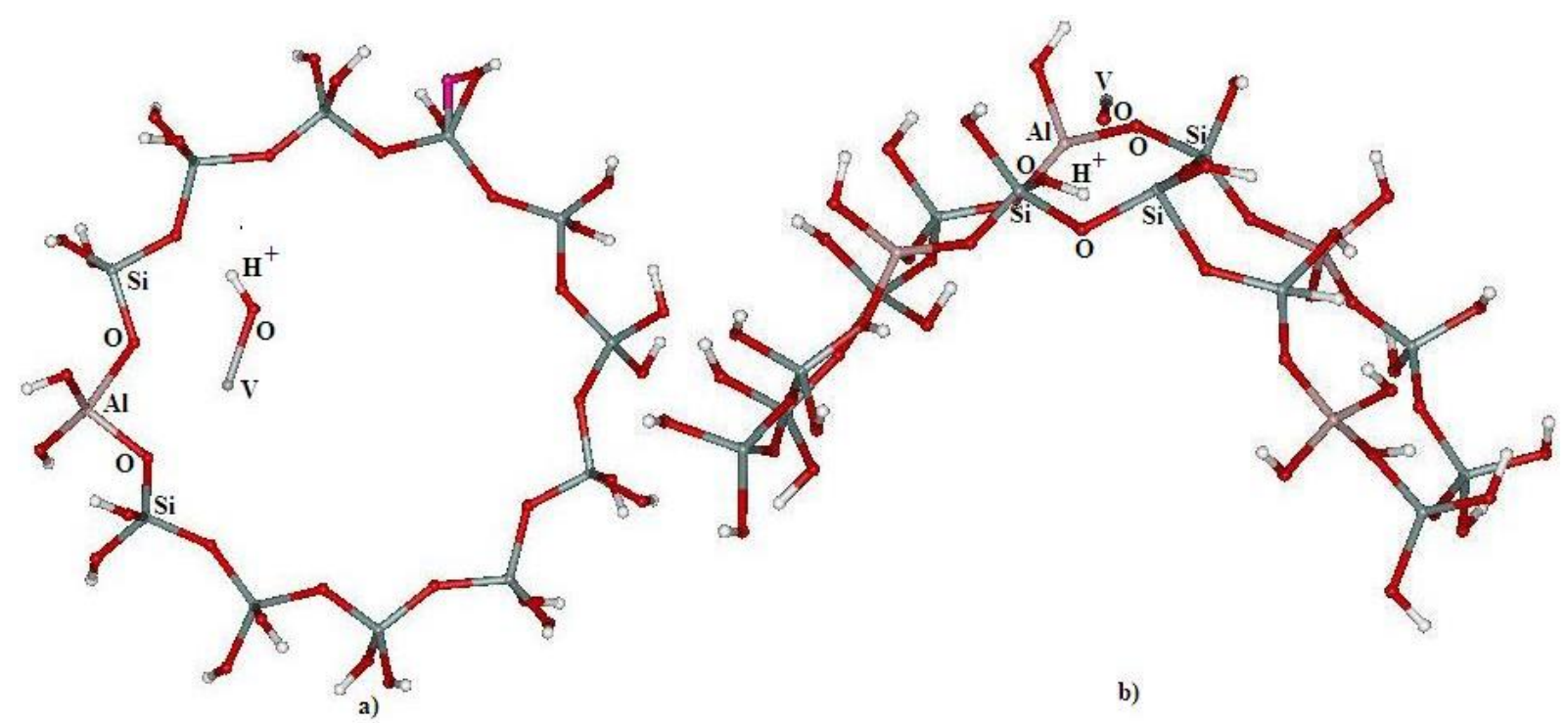

Figure 4. The final interchanges bond atoms after VO impact with HY-zeolite in two models are presented modification in the bonds, a) the bond breaking of $\mathrm{H}^{+} \mathrm{O}$ and new fragment of $\mathrm{VO}-\mathrm{H}^{+}$, b) the $\mathrm{VO}$ adsorption in 4 T-sites without bond breaking over $\mathrm{AlOH}^{+}$.

The analysis of results obtained for dynamics calculation for the first model found the change in the atomic positions of the acid site due to the presence of vanadium oxide. The first point considered was the speed values with 3.0 where the diffusion of VO moves along the ring without representative modification over atoms of acid site, this means that a net force is exerted by each nuclei of the acid site affecting VO. For this, there is a decrease of the speed value to 1.5 , it shows a slow movement and promotes an adsorption process of VO because the net force is representative attraction of the $\mathrm{VO}$ over acid site generating changes in the atomic bonds $\left(\mathrm{H}^{+} \mathrm{OAlO} 3\right)$, in adsorption process when (VO) interacts with the $(\mathrm{OH}+)$ has energy of $25 \mathrm{kcal} / \mathrm{mol}$ associated with the bond breaking of (O$\mathrm{H}^{+}$) after that this appended to (VO) with an energetic change of $28 \mathrm{kcal} / \mathrm{mol}$ to form bond (V-O$\mathrm{H}^{+}$) as shown in (Fig. 4) a). In another hand the analysis of dynamics calculation for the second model when VO molecule impacts on the surface nearly acid site with speed value of 3.0 the net force is totally repulsive. Taking into account, the speed was increased to 5.0 to overcome the potential barrier repulsive generated by the 4 tetrahedral sites then occur an attractive force on the acid site due to the presence of vanadium oxide producing modified bond length on $(\mathrm{H}+\mathrm{OAlO} 3)$. In the atomic modification the total energy is $40 \mathrm{kcal} / \mathrm{mol}$ that corresponds to bond length for the vanadium-oxygen
(V-O) and $\left(\mathrm{OH}^{+}\right)$, affirming that the adsorption process is obtained as shown in (Fig. 4) b).

\section{Conclusions}

Results allow doing the following conclusions: In both cases exist adsorption processes that take their initial condition in the speed and position. The physical environment of the oxygen atoms in the acid site is strongly affected by the presence of vanadium oxide. In the first case, major modified of bond length quantity is taken by tetrahedral $\mathrm{Al}$ and $\mathrm{Si}$ when vanadium oxide is bonded to the acid site with hydrogen extracted and elucidating the activity loss. Those changes in bond length atoms are presented inside of zeolite ring all changes in the length of bonds as consequence of confinement effect due to of ring form.

In the case of zeolite surface the VO only presents an attraction force nearly over the acid site like adsorption process with an important modification of $\mathrm{VO}$ atomic bonds. It has been interesting to find atomic bond modification for the changes in the atomics bond length presented within the two models of the zeolite and affect differently. It has been interesting to elucidate the loss of activity by the atomic modification and by the interaction between HY-zeolite and VO with dynamics aspects. 


\section{Acknowledgements}

I.P. Zaragoza thankfully acknowledge the computer resources, technical expertise and support provided by the Laboratorio Nacional de Supercómputo del Sureste de México through the grant number P2016/007 and to Patricia Galicia.

\section{References}

1- R.H. Harding, A.W. Peters, J.R.D. Nee New developments in FCC catalyst technology. Appl Catal A: General. 2001, Dec; 221(1), 389-396.

2- P.I. Premovic, D.M. Dordevic, M.S. Pavlovic. Vanadium of petroleum asphaltens and source kerogens. Fuel. 2002, Oct; 81(15), 2009-2016.

3- J. Haber, M. Witko, and R. Tokarz. Vanadium pentoxide I. Structures and properties. Appl Catal A General. 1997, Sept; 157(1), 3-22.

4- R. Dimitrova, Y. Neinska, M. Mihályi, G. PalBorbély, M. Spassova. Reductive solid-state ion exchange as a way to vanadium introduction in BZSM and BBeta zeolites. App Catal A: General. 2004, Mar; 266(1), 123-127.

5- L.A. Pine. Vanadium-catalyzed desrtruction of USY zeolite. J. Catal. 1990, Sept; 125(2), 514-524.

6- M. Xu, X. Liu, R.J. Madon. Pathway for Yzeolite destruction: the rol of sodium and vanadium. J Catal. 2002, Apr; 207(2), 237-246.

7- H.S. Cerqueira, G. Caeirob, L. Costac, F.R. Ribeiroc, Deactivation of FCC catalysts. J. Mol Catal A, Chemical. 2008 Sept; 292(1), 1-13.

8- U.J. Etim, B. Xu, R. Ullah, Z. Yan. Effect of vanadium contamination on the framework and micropore structure of ultra-stable Y-zeolite. J. Colloid Interface Sci., 2016, Oct; 463,188-198.

9- J.B. Parise, D.R. Corbin, L. Abrams, D.E. Cox. Structure of dealuminated lindeY-zeolite. Acta Cryst. 1984, May; C40(1), 1493-1497.

10-M. Niwa, K. Susuki, K. Isamoto, N. Katada. Identification and measurement of strong bronsted acid side in ultrastable Y-zeolite. J. Phys Chem B. 2006, Oct; 110, 264-269.

11-P.J. Hay, A.Y. Redondo Guo. Theoretical studies of pentene cracking on zeolite: c-c $\beta$-scission process. Catal Today. 1999;50, 517-523.

12-I.P. Zaragoza, L.A. Garcia-Serrano, R. Santamaria. Selectivity of a model zeolite ring over hydrocarbons with different symmetry travelling with different orientations and speeds. J. Phys Chem B. 2005, Dec; 109,705.

13-R.G. Parr, W. Yang. Density functional theory of atoms and molecules. Oxford University Press: New York 1989. 333 p.

14-S.M. Valiev, E.J. Bylaska, N. Govind, K. Kowalski, T.P. Straatsma, H.J.J. Van Dam, D. Wang, D.J. Nieplocha, E. Apra, T.L. Windus, W.A. de Jong. N.W.Chem: A comprehensive and scalable open-source solution for large scale molecular simulations. Comput Phys Commun. 2010, Sept: 181(9), 1477-1489.

15-A.D. Becke. Density-functional exchange-energy approximation with correct asymptotic behavior. Phys Rev A. 1988 Sept; 38, 3098.

16-C. Lee, W. Yang, R.G. Parr. Development of the Colle-Salvetti correlation-energy formula into a functional of the electron density. Phys Rev B. 1988, Jan; 37, 785.

17-A . Ramírez-Solís . On the performance of local, semilocal, and nonlocal exchange-correlation functionals on transition metal molecules. J. Chem Phys. 2007, Jun; 126(22), 224105.

18-P.J. Hasnip, K. Refson, M.I.J. Probert, J.R. Yates, S.J. Clark, C.J. Pickard. Density functional theory in the solid state. Phil Trans R Soc A. 2014, Feb; 372,1-26.

19. I.P. Zaragoza, J.H. Pacheco-Sánchez, I. Echevarria-Chan, A. Bravo-Ortega. DFT study of interaction between a hydrogen molecule and AgY-zeolite. Rev Mex Fis. 2014, Nov; 60(6), 460-465. 